\title{
Customized Adjuvant Chemotherapy Based on Biomarker Examination May Improve Survival of Patients Completely Resected for Non-small-cell Lung Cancer
}

\author{
DAGE LIU, NARIYASU NAKASHIMA, JUN NAKANO, SHINTARO TARUMI, NATSUMI MATSUURA, TAKAYUKI \\ NAKANO, KAZUHITO NII, YOSHIMASA TOKUNAGA, TETSUHIKO GO and HIROYASU YOKOMISE \\ Department of General Thoracic Surgery, Faculty of Medicine, Kagawa University, Takamatsu, Japan
}

\begin{abstract}
Aim: Adjuvant platinum-based chemotherapy is recommended for patients with completely resected stage II (N1) or III (N2) non-small cell lung cancer (NSCLC). However, the optimal chemotherapy regimen is difficult to predict for individual patients. Our previous prospective study on individualized treatment according to biomarker status, such as excision repair cross-complementing 1 (ERCC1), class III $\beta$-tubulin (tubulin), thymidylate synthase (TYMS) and ribonucleotide reductase M1 (RRM1), achieved encouraging results in patients with advanced NSCLC. The present study further examined the effect of biomarker-based adjuvant chemotherapy in patients with completely resected NSCLC. Patients and Methods: Between January 2006 and December 2014, 66 patients with localized (stage I-IIIA) NSCLC who underwent $R O$ operation received 2-4 cycles of platinum doublet adjuvant chemotherapy: Platinum plus docetaxel, platinum plus pemetrexed for adenocarcinoma, and platinum plus tegafur/gimeracil/oteracil combination (TS-1) for squamous cell carcinoma (SCC) were selected according to the registered protocol at each period. Immunohistochemistry was used to evaluate the biomarkers: ERCC1 status for platinum, tubulin for docetaxel, and TYMS for pemetrexed and TS-1. A matched chemotherapy regimen meant that platinum plus docetaxel was administered in patients negative for ERCC1 and negative for tubulin, platinum plus pemetrexed in patients with adenocarcinoma positive for tubulin, negative for ERCC1 and negative for TYMS, and platinum plus TS-1 in those with SCC positive for tubulin, negative for ERCCI and negative for TYMS. Results: The 5-year survival rate was
\end{abstract}

Correspondence to: Dage Liu, MD, Department of General Thoracic Surgery, Faculty of Medicine, Kagawa University, 17501 Ikenobe, Miki-cho, Kita-gun, Kagawa 761-0793, Japan. Tel: +81 878912191, Fax: +81 878912192, e-mail: dgliu@kms.ac.jp

Key Words: Adjuvant chemotherapy, lung cancer surgery, cancer biology, survival, biomarker.
$77.5 \%$ considering all 66 patients, and $85.7 \%, 71.8 \%$, and $78.8 \%$ for those with p-stage I, II, and III, respectively. Patients who received a matched chemotherapy regimen ( $n=13$; platinum plus docetaxel in eight, platinum plus pemetrexed in five) had significantly better 5-year survival than patients with unmatched biomarker status $(n=53)(100 \%$ vs. $71.0 \%, p=0.0011)$. Conclusion: Customized adjuvant chemotherapy based on biomarker examination significantly improved the survival of patients with NSCLC, regardless of p-stage.

Up to $60 \%$ of patients with localized (stage I-IIIA) non-small cell lung cancer (NSCLC) develop recurrence even after complete resection (1), The 5-year survival rates without additional treatment have been found to range from $23 \%$ in patients with stage IIIA disease to $67 \%$ in those with stage IA disease (2). Adjuvant platinum-based chemotherapy is recommended for patients with completely resected stage II (N1) or III (N2) NSCLC $(3,4)$ and for those with stage I NSCLC with blood vessel or lymphatic invasion, which is an indicator of poor prognosis. Based on a recent meta-analysis of five cisplatin-based trials, adjuvant chemotherapy was found to increase the 5-year survival rate by $5.4 \%$ (5).

Further improvement in outcome of adjuvant chemotherapy may be expected from its customization. However, the optimal chemotherapy regimen for individual patients is difficult to predict. Recently, several biomarkers were shown to be associated with responsiveness to chemotherapy (6-11). In addition, we reported that low expression of excision repair cross-complementing 1 (ERCC1), class III $\beta$-tubulin (tubulin), thymidylate synthase (TYMS), and ribonucleotide reductase M1 (RRM1) was indicative of favorable prognosis in patients with c-N2 and c-N3 NSCLC treated with surgery after induction chemoradiotherapy (12). Moreover, administration of customized induction chemotherapy according to ERCC1, tubulin, TYMS, and RRM1 status achieved encouraging results in patients with advanced NSCLC (13). In the present study, we aimed to prospectively explore the effects of 
Table I. Patient and treatmnet characteristics.

\begin{tabular}{lcc}
\hline Characteristic & No. of patients & Percentage \\
\hline Total no. of patients & 66 & 100 \\
Age, years & & \\
$\quad$ Median & 63.2 & \\
$\quad$ Range & $34-78$ & \\
Gender & & \\
$\quad$ Male & 46 & 84.0 \\
Female & 20 & 16.0 \\
Histology & & \\
Adenocarcinoma & 49 & 74.2 \\
Squamous cell carcinoma & 15 & 22.7 \\
Other & 2 & 3.1 \\
Clinical stage & & \\
I & 12 & 18.2 \\
II & 24 & 36.4 \\
IV & 30 & 45.4 \\
Therapeutic menu & & \\
Platinum + DOC & 30 & 45.5 \\
Platinum + PEM & 25 & 37.8 \\
Platinum + S-1 & 5 & 7.6 \\
Platinum + VNR & 5 & 1.5 \\
Platinum + GEM & 1 & \\
\hline
\end{tabular}

DOC: Docetaxel; PEM: pemetrexed; S-1: TS-1, the combination drug tegafur/gimeracil/oteracil; GEM: gemcitabin; VNR: vinorebine.

adjuvant chemotherapy, customized according to the status of these biomarkers, in patients with completely resected NSCLC.

\section{Patients and Methods}

Patients and treatments. This study was approved by the Institutional Review Board of Kagawa University, and written informed consent was obtained from all patients. Between January 2006 and December 2014, 66 patients with pathologically proven NSCLC were enrolled. These patients included 46 males and 20 females; 49 patients had adenocarcinoma, 15 had squamous cell carcinoma, and two had other carcinoma types (Table I). Patients with pathological stage I (with vessel or lymphatic invasion), II (N1), or IIIA (N2) disease underwent an R0 operation and received a platinum doublet adjuvant chemotherapy (2-4 cycles).

The intratumoral expression of ERCC1, tubulin, TYMS and RRM1 were measured by immunohistochemistry as reported previously (12). After biomarker examination, chemotherapy was basically selected according to biomarker status. The criteria used for drug selection is presented in Figure 1 as reported previously (13), using ERCC1 status for platinum; tubulin for docetaxel; and TYMS for pemetrexed and tegafur/gimeracil/oteracil combination (TS-1). As a result, platinum doublets were selected according to the registered protocol at each period (Figure 1).

All patients underwent adjuvant chemotherapy for two to four cycles (12). Briefly, this regimen consisted of carboplatin at an area under the curve of $6 \mathrm{mg} / \mathrm{ml} \mathrm{min} \mathrm{(30-min} \mathrm{intravenous} \mathrm{infusion),}$ docetaxel at $60 \mathrm{mg} / \mathrm{m}^{2}$ (3-h intravenous infusion), pemetrexed at

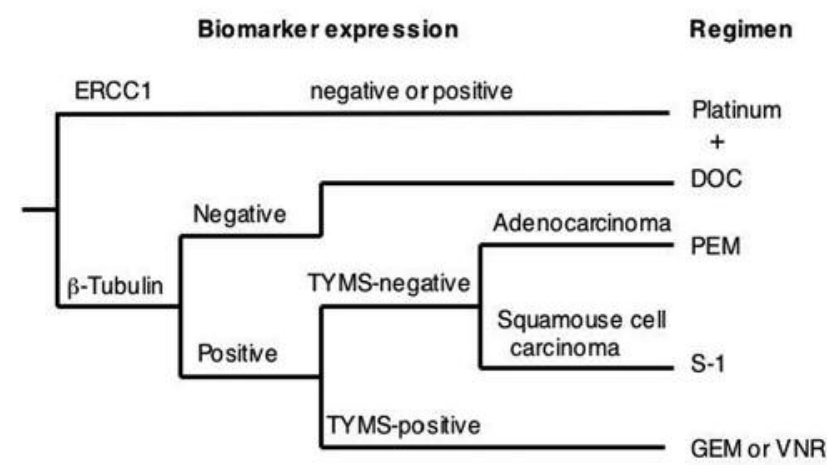

Figure 1. The criteria used for drug selection in the present study. ERCC1: Excision repair cross-complementing 1; $\beta$-Tubulin: class III $\beta$-tubulin; TYMS: thymidylate synthase; DOC: docetaxel; PEM: pemetrexed; TS-1: tegafur/gimeracil/oteracil; GEM: gemcitabine; VNR: vinorelbine.

$500 \mathrm{mg} / \mathrm{m}^{2}$ on day 1 at weeks $1,2,3$, and 5 plus concurrent thoracic irradiation at a dose of $50 \mathrm{~Gy}$. For the carboplatin and TS-1 arm, TS- 1 at $80 \mathrm{mg} / \mathrm{m}^{2}$ was given from day 1 to day 15 in every cycle.

Immunohistochemistry. Immunohistochemistry was performed on a 4- $\mu \mathrm{m}$ sections of formalin-fixed paraffin-embedded tissue as reported previously $(10,12,13)$ (Figure 2). A mouse monoclonal antibody against ERCC1 (diluted 1:200; FL-297; Santa Cruz Biotechnology, Inc., Santa Cruz, CA, USA), a rabbit monoclonal antibody against tubulin (diluted 1:500; EP1569Y; Epitomics, Inc., Burlingame, CA, USA), a rabbit monoclonal antibody against TYMS (diluted 1:500; kindly provided by Dr. M. Fukushima, Tokushima Research Center, Japan), and a rabbit polyclonal antibody against RRM1 (diluted 1:500; 10526-1-AP; Protein Tech Group, Chicago, IL, USA) were used. All immunostained sections were independently evaluated by two investigators who had no knowledge of the patient characteristics. A sample was classified as: ERCC1-positive tumor if $>30 \%$ of the tumor cells exhibited positive staining; tubulin-positive tumor if $>30 \%$ of the tumor cells were positively stained for tubulin; RRM1-positive tumor if $>40 \%$ of the tumor cells were positively stained for RRM1. For evaluation of TYMS, all sections were scored in a semiquantitative manner according to a method described previously (12). The HSCORE was obtained for each slide using the following algorithm: HSCORE= $\Sigma(\mathrm{I} \times \mathrm{PC})$, where I and PC represent the staining intensity and the percentage of cells staining at each intensity respectively. If the HSCORE for TYMS in a given specimen was $>30$, the sample was classified as TYMS-positive.

Statistical analysis. Overall survival was defined as the time from treatment initiation to the date of death due to any cause. Diseasefree survival was defined as the time from treatment initiation to the date of relapse. The Kaplan-Meier method was used to estimate the probability of overall and disease-free survival as a function of time, and differences in the survival of patient subgroups were compared using Mantel's log-rank test. All $p$-values were based on two-tailed statistical analysis, and differences at $p<0.05$ were considered to be statistically significant. 

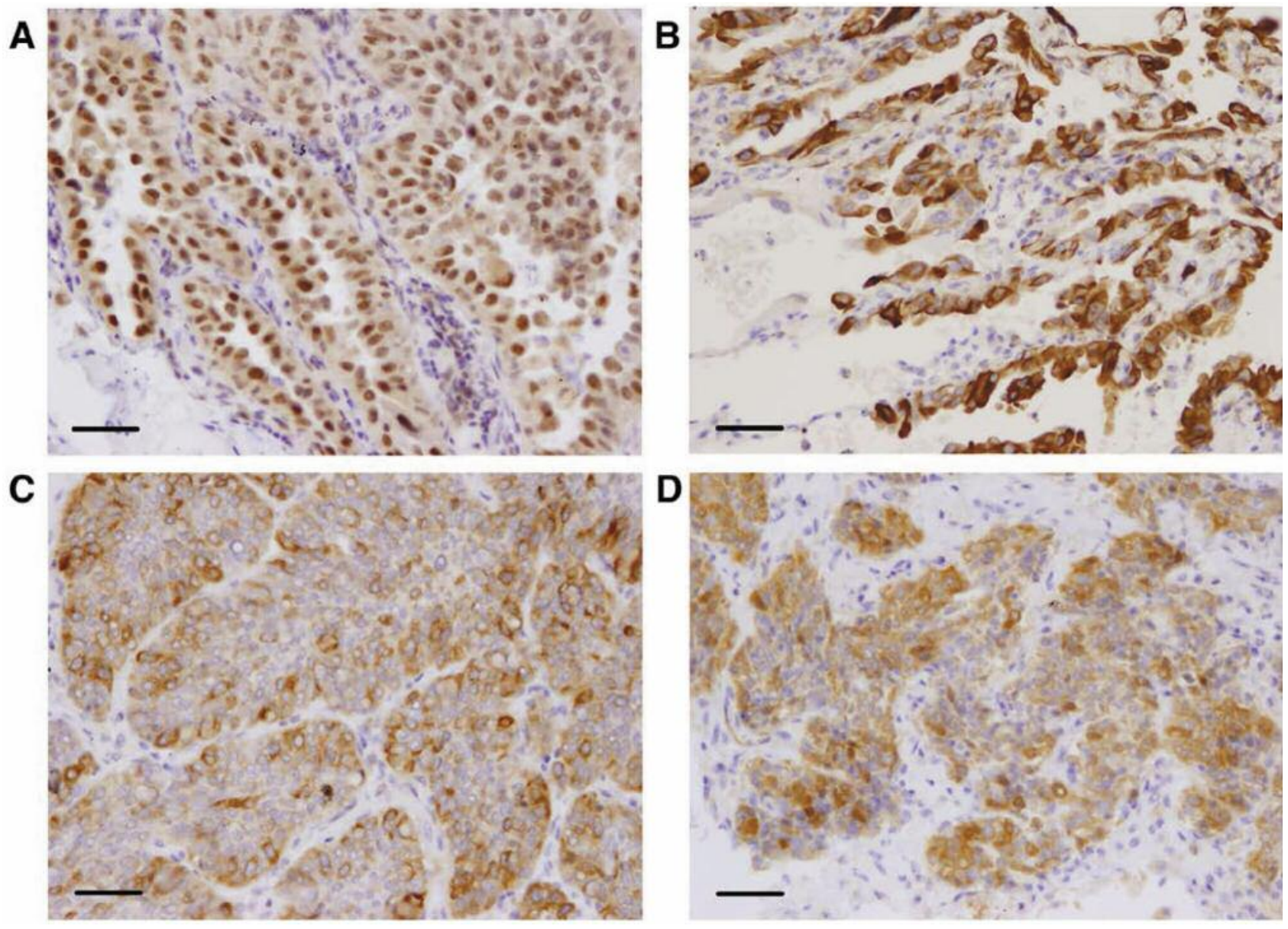

Figure 2. Immunohistochemical staining of biomarkers in lung cancer. Representative carcinoma with positive expression of excision repair crosscomplementing $1(A)$, class III $\beta$-tubulin (B), ribonucleotide reductase $M 1(C)$ and thymidylate synthase (D). Bars: $50 \mu \mathrm{m}$.

\section{Results}

Biomarker status. ERCC1 expression: Intratumoral ERCC1 expression was confined to the nucleus (Figure 2A). The percentage of ERCC1-positive tumor cells varied greatly (median $=50.0 \% ; \quad$ mean $\pm \mathrm{SD}=44.5 \pm 23.1 \%$ ). Among 66 patients, $39(59.1 \%)$ were ERCC1-positive (Table II). No significant relation was observed between the ERCC1 status and patient variables, such as sex, tumor status, nodal status, clinical stage and histology (Table II).

$\beta$-Tubulin expression: Intratumoral $\beta$-tubulin expression exhibited cytoplasmic staining pattern (Figure 2B). The percentage of tumor cells positively stained for tubulin varied greatly (median $=57.5 \%$; mean $\pm \mathrm{SD}=51.0 \pm 28.9 \%$ ). Among 66 patient with NSCLCs, 46 (69.7\%) were tubulinpositive. Tubulin-positive tumors accounted for 12 out of 24 adenocarcinomas $(50.0 \%)$ and seven out of the 21 squamous cell carcinomas (33.3\%). No significant relation was observed between tubulin status and patient variables (Table II).

RRM1 expression: Intratumoral RRM1 expression exhibited a cytoplasmic staining pattern (Figure 2C). The percentage of RRM1-positive tumor cells varied greatly (median $=50.0 \%$; mean $\pm \mathrm{SD}=44.3 \pm 26.3 \%$ ). Among 66 NSCLC patients, 44 tumors (66.7\%) were RRM1-positive (Table II). No significant relation was observed between RRM1 status and the patient variables (Table II).

TYMS expression: Intratumoral TYMS expression exhibited a cytoplasmic staining pattern (Figure 2D). The percentage of TYMS-positive tumor cells varied greatly (median=60.0; mean $\pm \mathrm{SD}=60.2 \pm 35.2$ ). Among 66 patients with NSCLCs, 24 (36.4\%) were TYMS-positive (Table II). No significant relation was observed between the TYMS status and patient variables (Table II). 
Table II. Distribution of 66 patients with non-small cell lung cancer according to biomarkers' status.

\begin{tabular}{|c|c|c|c|c|c|c|c|c|c|c|c|c|c|}
\hline \multirow[b]{2}{*}{ Variable } & \multirow[b]{2}{*}{ (n) } & \multicolumn{3}{|c|}{ ERCC1 } & \multicolumn{3}{|c|}{$\beta$-Tubulin } & \multicolumn{3}{|c|}{ TYMS } & \multicolumn{3}{|c|}{ RRM1 } \\
\hline & & Negative & Positive & $p$-Value & Negative & Positive & $p$-Value & Negative & Positive & $p$-Value & Negative & Positive & $p$-Value \\
\hline \multicolumn{14}{|l|}{ Gender } \\
\hline Male & 46 & 21 & 25 & 0.2346 & 15 & 31 & 0.5365 & 16 & 30 & 0.6855 & 15 & 31 & 0.8498 \\
\hline Female & 20 & 6 & 14 & & 5 & 15 & & 8 & 12 & & 7 & 13 & \\
\hline \multicolumn{14}{|c|}{ Pathologic tumor status } \\
\hline $\mathrm{T} 1$ & 23 & 4 & 19 & 0.0072 & 5 & 18 & 0.4424 & 8 & 15 & 0.9789 & 5 & 18 & 0.3325 \\
\hline $\mathrm{T} 2$ & 32 & 19 & 13 & & 12 & 20 & & 12 & 20 & & 13 & 19 & \\
\hline $\mathrm{T} 3, \mathrm{~T} 4$ & 11 & 4 & 7 & & 3 & 28 & & 4 & 7 & & 4 & 7 & \\
\hline \multicolumn{14}{|c|}{ Pathologic nodal status } \\
\hline N0,1 & 38 & 16 & 22 & 0.8179 & 12 & 26 & 0.7927 & 12 & 26 & 0.3465 & 9 & 29 & 0.0527 \\
\hline $\mathrm{N} 2,3$ & 28 & 11 & 17 & & 8 & 20 & & 12 & 16 & & 13 & 13 & \\
\hline \multicolumn{14}{|l|}{ Pathologic stage } \\
\hline I & 12 & 5 & 7 & 0.9906 & 1 & 11 & 0.1218 & 4 & 8 & 0.2901 & 3 & 9 & 0.2901 \\
\hline II & 24 & 10 & 14 & & 10 & 14 & & $7 a ̊$ & 17 & & 6 & 18 & \\
\hline III & 30 & 12 & 18 & & 9 & 21 & & 13 & 17 & & 13 & 17 & \\
\hline \multicolumn{14}{|l|}{ Histology } \\
\hline Adenocarcinoma & 49 & 20 & 29 & 0.1974 & 13 & 36 & 0.5053 & 20 & 29 & 0.3141 & 17 & 32 & 0.7442 \\
\hline $\mathrm{Sq}$ & 15 & 5 & 10 & & 6 & 9 & & 3 & 12 & & 4 & 11 & \\
\hline Other & 2 & 2 & 0 & & 1 & 1 & & 1 & 1 & & 1 & 1 & \\
\hline Total & 66 & 27 & 39 & & 20 & 46 & & 24 & 42 & & 22 & 44 & \\
\hline
\end{tabular}

ERCC1: Excision repair cross-complementing $1 ; \beta$-tubulin: class III $\beta$-tubulin, RRM1: ribonucleotide reductase M1; TYMS: thymidylate synthase; Sq: squamous cell carcinoma.

Correlations among ERCC1, tubulin, RRMI and TYMS expression. No correlation was observed between ERCC1 and tubulin expression $(\mathrm{r}=0.085, p=0.4988)$. Significant positive correlations were observed between ERCC1 and TYMS expression ( $\mathrm{r}=0.347, p=0.0043)$, ERCC1 and RRM1 expression ( $\mathrm{r}=0.354, p=0.0035)$, and RRM1 and TYMS expression $(\mathrm{r}=0.603, p<0.0001)$. On the other hand, no correlation was observed between tubulin and TYMS expression ( $\mathrm{r}=0.117, p=0.3476)$ or RRM1 expression $(\mathrm{r}=0.021, p=0.8644)$. Moreover, among the 66 tumors, only four $(4.1 \%)$ exhibited negative expression of ERCC1, tubulin, TYMS and RRM1; 20 (30.3\%) exhibited positive expression of all four biomarkers; and 42 (63.6\%) exhibited positive expression of at least one of the four biomarkers.

Regimen of adjuvant chemotherapy. Surgery was performed via open thoracotomy or thoracoscopy, and systematic mediastinal lymph node dissection was performed. There was no chemotherapy-associated mortality recorded in this study. Docetaxel $(n=30)$, pemetrexed $(n=25)$, TS-1 $(n=5)$, vinorelbine $(n=5)$, and gemcitabine $(n=2)$, were selected for adjuvant chemotherapy on the basis of biomarker status. In regard to biomarker status, $13(19.7 \%)$ patients received a completely matched chemotherapy regimen (platinumdocetaxel in eight, platinum-pemetrexed in five; stage II in 6 , stage III in 7$)$ and $53(80.3 \%)$ received a unmatched regimen (stage I in 12, stage II in 18, stage III in 23).
Disease-free and overall survival. The 5-year overall survival rate was $77.5 \%$ considering all 66 patients (Figure 3A). Patients who received a completely matched adjuvant chemotherapy $(n=13)$ had a significantly better survival rate than those who received an unmatched regimen $(n=53$ : stage I/II/III, 12/18/23) (5-year survival: $100 \%$ and $71.0 \%$, respectively; $p=0.0011$, Figure $3 \mathrm{~B}$ ). Regarding the clinical background, the 5-year survival rate was $85.7 \%, 71.8 \%$, and $78.8 \%$ for those with p-stage I, II, and III, respectively (Figure 3C). There was no significant correlation between survival and other clinical factors, such as age, gender and histology.

Thus far, 24 out of 66 patients have developed postoperative recurrence (eight at the primary site, seven with lung metastasis, four with brain metastasis, five with bone metastasis and one with adrenal metastasis). The 5-year disease-free survival rate was $57.7 \%$ for the whole patient group, and $75.0 \%, 64.3 \%$ and $46.2 \%$ for those with p-stage I, II and III disease, respectively (Figure 4C). There was no significant correlation between the disease-free survival and other clinical factors, such as age, gender and histology.

\section{Discussion}

Various biomarkers have been described to predict response to cytotoxic agents. Sub-analysis of data from the International Adjuvant Lung Cancer Trial suggested that 

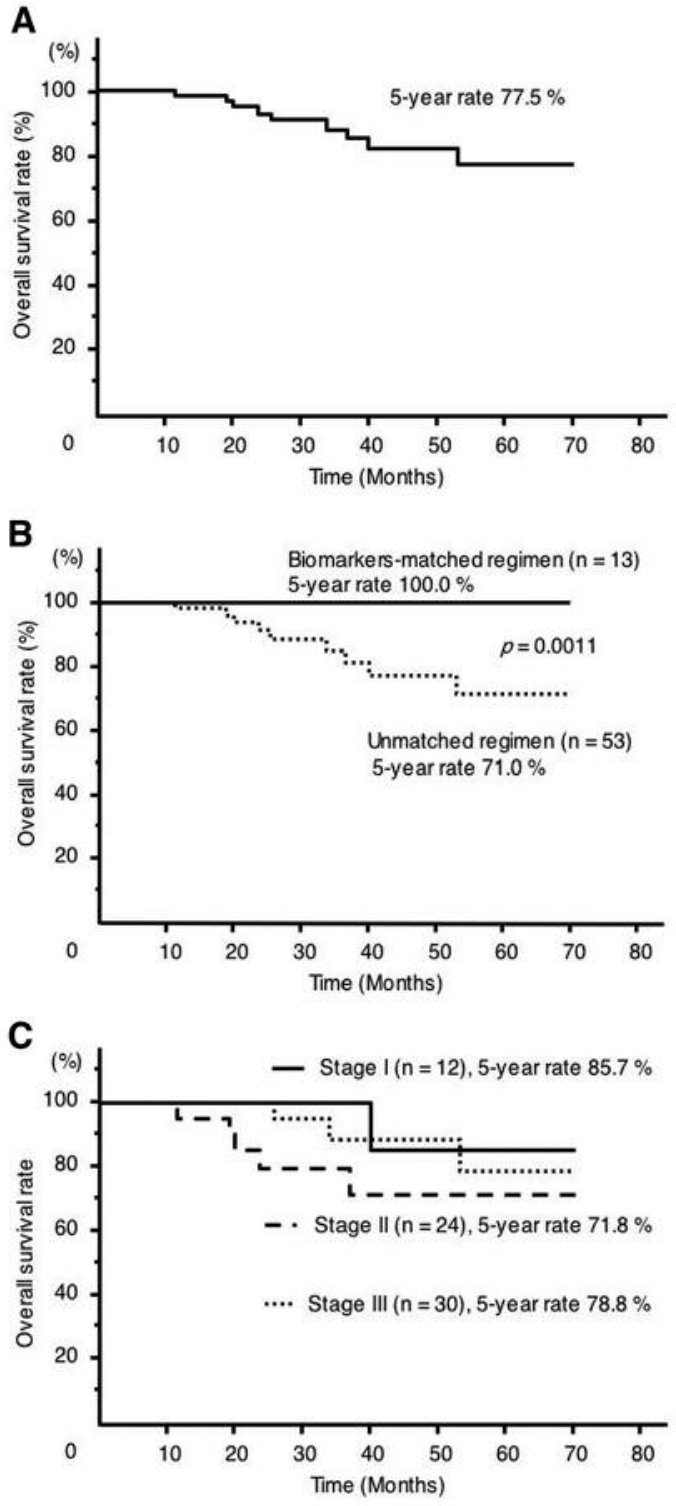

Figure 3. Overall survival for the whole cohort of patients with nonsmall cell lung cancer $(n=66)$ after adjuvant chemotherapy based on biomarker examination $(A)$, and in relation to biomarker status $(B)$ and pathological stage $(C)$.

ERCC1 was an important biomarker of response to platinum-based chemotherapy (9). Seve et al. reported that low expression of tubulin was associated with better survival in patients with advanced lung cancer treated with a paclitaxel-based regimen (7). The Spanish Lung Cancer Group reported that a low level of $R R M 1$ mRNA was associated with better survival in patients with advanced lung cancer who received a gemcitabine/cisplatin regimen (6). We also reported that low expression of TYMS was
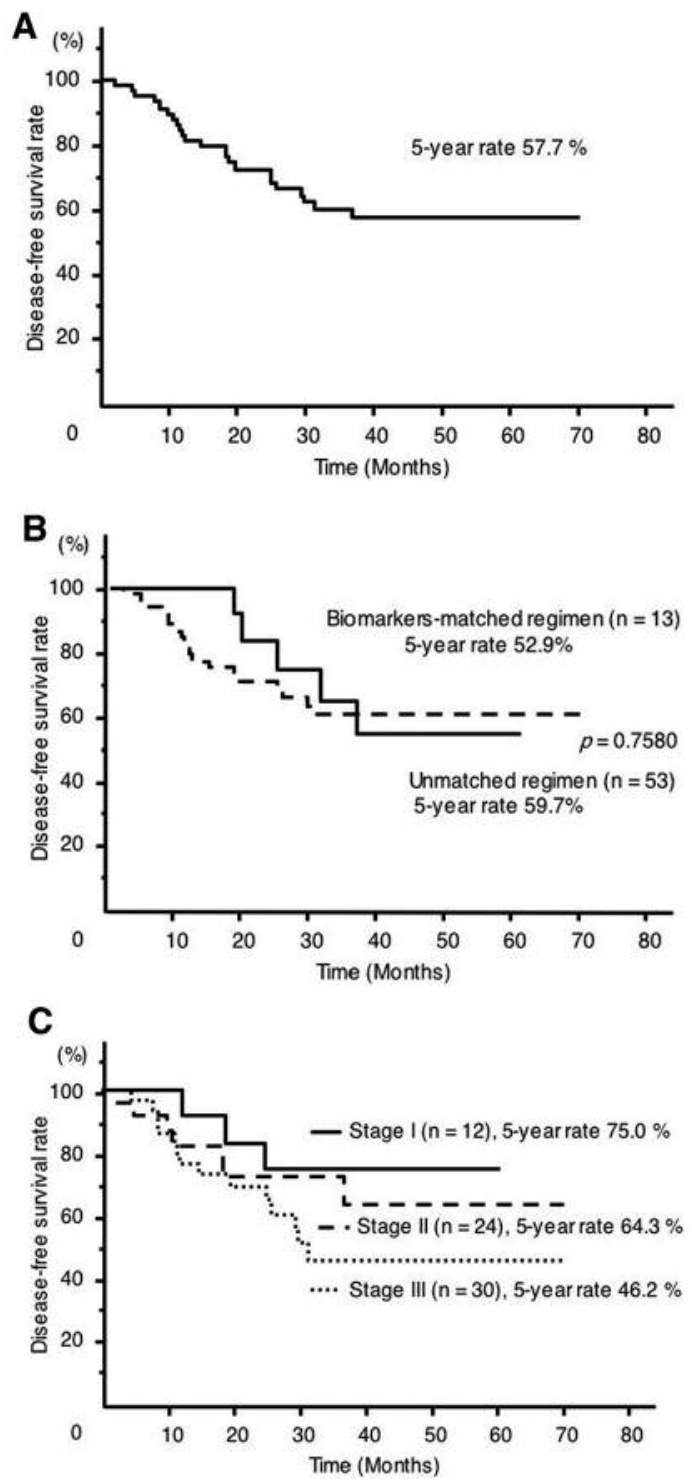

Figure 4. Disease-free survival for the whole cohort of patients with non-small cell lung cancer $(n=66)$ after adjuvant chemotherapy based on biomarker examination (A), in relation to biomarker-matched status (B) and pathological stage $(C)$.

associated with better survival in patients who received adjuvant chemotherapy with a tegafur/uracil-based regimen and that there was no correlation among RRM1, ERCC1 and tubulin expression $(8,12)$. Furthermore, pemetrexed and TS-1 were shown not to be inferior to first-line chemotherapy for advanced NSCLC $(14,15)$. Therefore, an appropriate chemotherapy can be selected for each individual on the basis of biomarker status, without any disadvantages in terms of outcomes. 
After receiving adjuvant chemotherapy that was customized according to the status of biomarkers, the 5-year overall survival rate for the whole cohort was $77.5 \%$ (Figure 3A). Patients who received biomarker-based adjuvant chemotherapy had significantly better survival compared to patients who received an unmatched regimen (100\% vs. $70 \%, p=0.0011$; Figure 3B). This demonstrates the benefit of biomarkers in improving the outcome of adjuvant chemotherapy for patients with NSCLC. Even in patients who received unmatched adjuvant chemotherapy (Figure $4 \mathrm{~A}$ ), the 5-year overall and disease-free survival rates were better than those previously reported $(2,5)$. In our previous study on biomarker-based induction chemotherapy, complete pathological or major response was achieved in $66.7 \%$ (14/21) and down-staging was observed in $76.2 \%$ of the study population. Furthermore, the 25 patients with stage IIIA to IV disease achieved an overall 5-year survival rate of $58.7 \%$; this rate increased to $73.6 \%$ for patients who underwent surgery (13).

In this present study, the 5-year survival rate was $85.7 \%$, $81.8 \%$, and $78.8 \%$ for $\mathrm{p}$-stage I, II, and III, respectively (Figure 3C). Regardless of p-stage, patients who received an adjuvant chemotherapy regimen matched by biomarker status had significantly better survival. Taken together, customized chemotherapy on the basis of biomarker examination was not only applicable to induction chemotherapy, but also to adjuvant chemotherapy for patients with a wide range of NSCLC stages.

Because ERCC1, that is a marker associated with response to platinum therapy, has been shown to have minimal changes in level of expression before and after chemotherapy compared with other biomarkers (12), platinum was thought to be a key cytotoxic agent and was included in all chemotherapy regimens. Although suitable biomarkers for patients with NSCLC remain unidentified, translational research has focused on characterizing several molecules for this purpose. In some studies that used only one or two biomarkers to customize chemotherapy $(7,16,17)$, the results were contradictory and patients had lower survival rates. In our series of studies, tubulin, RRM1 and TYMS were added to ERCC1 in order to increase the variation of available chemotherapy regimens. In the present study, platinum-docetaxel was used in $45.4 \%$ (30/66), platinumpemetrexed in $37.8 \%(25 / 66)$, platinum-TS-1 in $7.6 \%(5 / 66)$, platinum-vinorelbine in $7.6 \%(5 / 66)$, and platinumgemcitabine in $1.5 \%(1 / 66)$. This wide variety of choices enabled highly customized chemotherapy and resulted in encouraging outcomes.

This study had a number of limitations, particularly the small number of patients. The value of determining the biomarker status is inconsistent and controversial. The Canadian consensus recommendations on the use of biomarkers for treatment of NSCLC stated that there is currently insufficient evidence that testing for ERCC1, breast cancer susceptibility gene (BRCA), tubulin, RRM1, and p53 influences clinical treatment decisions (18). Booton et al. reported that ERCC1 mRNA levels were not associated with response and survival of patients with advanced NSCLC after platinum-based chemotherapy (19). Gomez-Roca et al. reported different expressions of biomarkers between primary NSCLC and lung metastases (20). Nevertheless, we deem it important to present our promising early results for patients with both early and advanced NSCLC. A second limitation was the dependence of the chemotherapy regimen on the status of the biomarkers. Although the four biomarkers that were used in this study have been reported to indicate the most common chemotherapy agents for NSCLC, only four (6.1\%) patients demonstrated negative expression of these biomarkers, whereas 20 (30.3\%) patients had positive expression. This implied that chemotherapy might not be sufficient in optimizing customized treatment of NSCLC. In addition to predictive biomarkers, the potential of targeted therapies, such as epidermal growth factor receptor-directed tyrosine kinase inhibitors (21), and tumor vaccines, such as MAGE-A3 (22), in improving the outcome of adjuvant chemotherapy for NSCLC needs to be further investigated.

In conclusion, our present prospective study on customized adjuvant chemotherapy according to ERCC1, tubulin, TYMS and RRM1 status achieved encouraging results in patients with completely resected NSCLC. The choice of chemotherapy regimen based on biomarker examination might be a useful approach for patients with both localized and advanced NSCLC.

\section{Conflicts of Interest}

None declared.

\section{Acknowledgements}

This work was partly supported by Grants-in-Aid for Scientific Research from the Japanese Society for the Promotion of Science, Grant no. 24390332 (HY) and Grant no. 22591435 (DL).

\section{References}

1 Brodowicz T, Ciuleanu T, Crawford J, Filipits M, Fischer JR, Georgoulias V, Gridelli C, Hirsch FR, Jassem J, Kosmidis P, Krzakowski M, Manegold C, Pujol JL, Stahel R, Thatcher N, Vansteenkiste J, Minichsdorfer C, Zochbauer-Muller S, Pirker R, Zielinski CC, Central European Cooperative Oncology G: Third CECOG consensus on the systemic treatment of nonsmall-cell lung cancer. Ann Oncol 23: 1223-1229, 2012.

2 Mountain CF: Revisions in the International System for Staging Lung Cancer. Chest 111: 1710-1717, 1997.

3 Arriagada R, Bergman B, Dunant A, Le Chevalier T, Pignon JP, Vansteenkiste $\mathrm{J}$; International Adjuvant Lung Cancer Trial 
Collaborative G: Cisplatin-based adjuvant chemotherapy in patients with completely resected non-small-cell lung cancer. $\mathrm{N}$ Engl J Med 350: 351-360, 2004.

4 Artal Cortes A, Calera Urquizu L and Hernando Cubero J: Adjuvant chemotherapy in non-small cell lung cancer: state-ofthe-art. Transl Lung Cancer Res 4: 191-197, 2015.

5 Pirker R: Adjuvant chemotherapy in patients with completely resected non-small cell lung cancer. Transl Lung Cancer Res 3: 305-310, 2014.

6 Rosell R, Danenberg KD, Alberola V, Bepler G, Sanchez JJ, Camps C, Provencio M, Isla D, Taron M, Diz P and Artal A: Ribonucleotide reductase messenger RNA expression and survival in gemcitabine/cisplatin-treated advanced non-small cell lung cancer patients. Clin Cancer Res 10: 1318-1325, 2004.

7 Seve P, Mackey J, Isaac S, Tredan O, Souquet PJ, Perol M, Lai $\mathrm{R}$, Voloch A and Dumontet C: Class III beta-tubulin expression in tumor cells predicts response and outcome in patients with non-small cell lung cancer receiving paclitaxel. Mol Cancer Ther 4: 2001-2007, 2005.

8 Nakano J, Huang C, Liu D, Masuya D, Nakashima T, Yokomise $\mathrm{H}$, Ueno $\mathrm{M}$, Wada $\mathrm{H}$ and Fukushima $\mathrm{M}$ : Evaluations of biomarkers associated with 5-FU sensitivity for non-small-cell lung cancer patients postoperatively treated with UFT. Br J Cancer 95: 607-615, 2006

9 Olaussen KA, Dunant A, Fouret P, Brambilla E, Andre F, Haddad V, Taranchon E, Filipits M, Pirker R, Popper HH, Stahel R, Sabatier L, Pignon JP, Tursz T, Le Chevalier T and Soria JC: DNA repair by ERCC1 in non-small-cell lung cancer and cisplatin-based adjuvant chemotherapy. N Engl J Med 355: 983-991, 2006.

10 Huang CL, Kadota K, Liu D, Ueno M, Nakasima N, Ishikawa $\mathrm{S}$, Gotoh M, Misaki N, Chang SS and Yokomise H: Expression of ERCC1 and class III beta-tubulin is associated with the survival of resected stage III non-small cell lung cancer patients treated with induction chemoradiotherapy using carboplatintaxane. Exp Ther Med 1: 445-451, 2010.

11 Sun JM, Han J, Ahn JS, Park K and Ahn MJ: Significance of thymidylate synthase and thyroid transcription factor 1 expression in patients with nonsquamous non-small cell lung cancer treated with pemetrexed-based chemotherapy. J Thorac Oncol 6: 1392-1399, 2011

12 Yokomise H, Liu D, Chang S, Go T, Ishikawa S, Misaki N and Nakashima N: Biomarkers as prognostic factors for $\mathrm{cN} 2$ or 3 nonsmall cell lung cancer treated by induction chemoradiotherapy and surgery. Anticancer Res 33: 1107-1115, 2013.

13 Yokomise H, Liu D, Ishikawa S, Go T, Gotoh M, Okuda M, Tarumi S, Kasai Y and Matsuura N: Chemotherapy followed by surgery on the basis of biomarker examination for patients with advanced non-small cell lung cancer. Anticancer Res 33: 55975602, 2013.

14 Scagliotti GV, Parikh P, von Pawel J, Biesma B, Vansteenkiste J, Manegold C, Serwatowski P, Gatzemeier U, Digumarti R, Zukin M, Lee JS, Mellemgaard A, Park K, Patil S, Rolski J, Goksel T, de Marinis F, Simms L, Sugarman KP and Gandara D: Phase III study comparing cisplatin plus gemcitabine with cisplatin plus pemetrexed in chemotherapy-naive patients with advanced-stage non-small-cell lung cancer. J Clin Oncol 26: 3543-3551, 2008.
15 Okamoto I, Yoshioka H, Morita S, Ando M, Takeda K, Seto T, Yamamoto N, Saka H, Asami K, Hirashima T, Kudoh S, Satouchi M, Ikeda N, Iwamoto Y, Sawa T, Miyazaki M, Tamura K, Kurata T, Fukuoka M and Nakagawa K: Phase III trial comparing oral S-1 plus carboplatin with paclitaxel plus carboplatin in chemotherapy-naive patients with advanced nonsmall-cell lung cancer: results of a west Japan oncology group study. J Clin Oncol 28: 5240-5246, 2010.

16 Gauthier I, Ding K, Winton T, Shepherd FA, Livingston R, Johnson DH, Rigas JR, Whitehead M, Graham B and Seymour L: Impact of hemoglobin levels on outcomes of adjuvant chemotherapy in resected non-small cell lung cancer: the JBR.10 trial experience. Lung Cancer 55: 357-363, 2007.

17 Tsao MS, Aviel-Ronen S and Ding K, Lau D, Liu N, Sakurada A, Whitehead M, Zhu CQ, Livingston R, Johnson DH, Rigas J, Seymour L, Winton $\mathrm{T}$ and Shepherd FA: Prognostic and predictive importance of $\mathrm{p} 53$ and RAS for adjuvant chemotherapy in non small-cell lung cancer. J Clin Oncol 25: 5240-5247, 2007.

18 Ellis PM, Blais N, Soulieres D, Ionescu DN, Kashyap M, Liu G, Melosky B, Reiman T, Romeo P, Shepherd FA, Tsao MS and Leighl NB: A systematic review and Canadian consensus recommendations on the use of biomarkers in the treatment of non-small cell lung cancer. J Thorac Oncol 6: 1379-1391, 2011.

19 Booton R, Ward T, Ashcroft L, Morris J, Heighway J and Thatcher N: ERCC1 mRNA expression is not associated with response and survival after platinum-based chemotherapy regimens in advanced non-small cell lung cancer. J Thorac Oncol 2: 902-906, 2007.

20 Gomez-Roca C and Raynaud CM, Penault-Llorca F, Mercier O, Commo F, Morat L, Sabatier L, Dartevelle P, Taranchon E, Besse B, Validire P, Italiano A and Soria JC: Differential expression of biomarkers in primary non-small cell lung cancer and metastatic sites. J Thorac Oncol 4: 1212-1220, 2009.

21 Pirker R, Minar W and Filipits M: Integrating epidermal growth factor receptor-targeted therapies into platinum-based chemotherapy regimens for newly diagnosed non-small-cell lung cancer. Clin Lung Cancer 9(Suppl 3): S109-115, 2008.

22 Ulloa-Montoya F and Louahed J, Dizier B, Gruselle O, Spiessens B, Lehmann FF, Suciu S, Kruit WH, Eggermont AM, Vansteenkiste $\mathrm{J}$ and Brichard VG: Predictive gene signature in MAGE-A3 antigen-specific cancer immunotherapy. J Clin Oncol 31: 2388-2395, 2013.
Received January 5, 2017

Revised March 1, 2017

Accepted March 31, 2017 